\title{
Title: Sustainable polyesters via direct functionalization of lignocellulosic sugars
}

Authors: Lorenz P. Manker ${ }^{1}$, Graham R. Dick ${ }^{1}$, Adrien Demongeot ${ }^{2}$, Maxime A. Hedou ${ }^{1}$, Christèle Rayroud $^{1}$, Thibault Rambert ${ }^{1}$, Marie J. Jones ${ }^{1,5}$, Irina Sulaeva ${ }^{3,4}$, Yves Leterrier ${ }^{2}$, Antje Potthast ${ }^{3}$, François Marechal ${ }^{5}$, Veronique Michaud ${ }^{2}$, Harm-Anton Klok ${ }^{6}$, and Jeremy S. Luterbacher ${ }^{*}$

\section{Affiliations:}

${ }^{1}$ Laboratory of Sustainable and Catalytic Processing (LPDC), Institute of Chemicals Sciences and Engineering (ISIC), School of Basic Sciences (SB), École Polytechnique Fédérale de Lausanne (EPFL); Lausanne, Switzerland.

${ }^{2}$ Laboratory for Processing of Advanced Composites (LPAC), Institute of Materials (IMX), School of Engineering (STI), École Polytechnique Fédérale de Lausanne (EPFL); Lausanne, Switzerland.

${ }^{3}$ Institute for Chemistry of Renewable Resources, Department of Chemistry, University of Natural Resources and Life Sciences; Wien, Austria.

${ }^{4}$ Wood K plus - Competence Center for Wood Composites \& Wood Chemistry, Kompetenzzentrum Holz GmbH; Linz, Austria.

${ }^{5}$ Industrial Energy Systems Laboratory, Ecole Polytechnique Fédérale de Lausanne; Lausanne, Switzerland.

${ }^{6}$ Institut des Matériaux and Institut des Sciences et Ingénierie Chimiques, Laboratoire des Polymères, École Polytechnique Fédérale de Lausanne (EPFL), Bâtiment MXD, Station 12, CH-1015 Lausanne, Switzerland.

*Corresponding author. E-mail: jeremy.luterbacher@epfl.ch

Abstract: The development of sustainable plastics from abundant renewable feedstocks has been limited by the complexity and efficiency of their production as well as their lack of competitive material properties. Here, we demonstrate the direct transformation of the hemicellulosic fraction of non-edible biomass into a diester plastic precursor at $83 \%$ yield ( $95 \%$ from commercial xylose) during integrated plant fractionation with glyoxylic acid. Melt polycondensation of the resulting xylose-based diester with a range of aliphatic diols led to high-molecular weight amorphous polyesters with combined high glass transition temperatures, tough mechanical properties, and strong gas barriers, which could be processed by injection-molding, thermoforming, and 3D-printing. These polyesters could then be chemically recycled from mixed plastic waste streams or digested under biologically relevant 
conditions. The transformation's simplicity led to projected costs that were competitive with fossil alternatives and significantly reduced associated greenhouse gas emissions, especially if glyoxylic acid was sourced from $\mathrm{CO}_{2}$.

\section{Introduction}

Biodegradable and recyclable biomass-derived polymers could facilitate our economies' decoupling from fossil resources and prevent the accumulation of plastics in the environment (1). However, producing these plastics from biomass at costs that are competitive with their fossil counterparts while achieving comparable material properties - namely similar thermal stability, mechanical strength, processability, and compatibility - has proven challenging $(2$, 3). Currently, most commercial bioplastics rely on efficient microbial fermentation of sugars (e.g., poly(lactic acid) [PLA], poly(hydroxyalkanoate)s [PHAs], poly(butylene succinate) [PBS]). These industrial fermentations constrain monomer production to linear aliphatic diacids, diols, and hydroxy acids, which has so far led to bioplastics with low glass transition temperatures $\left(\mathrm{T}_{\mathrm{g}}\right)$, poor ductility, or high gas permeability (4). When combined with high production costs from renewable resources, the lack of well-rounded material properties has made it difficult for bioplastics to compete with petroleum-based alternatives in a very competitive industry.

Alternatively, chemical sugar transformations have been proposed to access molecules with improved structure, notably rigid cyclic molecules that can increase $\mathrm{T}_{\mathrm{g}}$ and lower gas permeability. Rigid diacids such as 2,5-furandicarboxylic acid (FDCA), or rigid diols such as isosorbide (5) and acetalized sugar alcohols (6) can be polymerized to polymers with higher $\mathrm{T}_{\mathrm{g}}$. However, all these examples involve multi-step reaction sequences that require solvents, catalysts, or stoichiometric reagents. These steps increase production costs from sugars and reduce the overall yield. 
Currently, the sugars required for both fermentation and chemical processes are usually extracted from edible biomass, but ideally would be produced by depolymerization of lignocellulosic biomass's structural carbohydrates. Cellulose and hemicellulose are more abundant, can be grown on marginal lands, and their simple sugars are projected to be far less expensive and more sustainable as compared to their edible counterparts $(7,8)$. However, their industrial use has been limited by the high cost of their saccharification and subsequent fermentation, and the lack of mature integrated lignocellulosic conversion processes that can valorize the non-polysaccharide fractions of the plant, which notably include lignin (15-30 $\mathrm{wt} / \mathrm{wt} \%$ of lignocellulosic biomass). Promising bioplastics from non-carbohydrate plant fractions such as plant oils (9) or lignin (10) are emerging, but they are inherently limited by lower natural abundances - or, in the case of lignin, the lack of mature upgrading technologies - which makes achieving cost-competitiveness even more challenging than with sugars.

Therefore, producing inexpensive sustainable bioplastics with attractive structural and end-oflife properties will likely depend on developing simple and direct high-yielding routes from abundant renewable resources. Largely retaining the carbohydrate heterocycle in the final product structure could provide the required structural rigidity for both high $\mathrm{T}_{\mathrm{g}}$ and strong barrier properties, while at the same time ensuring high-atom economy and limiting the number of reactions. Recently, we developed a strategy that uses aldehydes to stabilize reactive intermediates during the fractionation of lignocellulosic biomass into an uncondensed, benchstable, acetal-stabilized lignin; highly-digestible, cellulose-rich solids; and acetal-stabilized xyloses (11-13). Here, we show that by using aldehydes with a secondary functionality, we can not only stabilize the hemicellulose-derived xylose and lignin, but also directly produce fused, heterotricyclic, difunctional monomers with unique characteristics for bioplastic production. Specifically, we demonstrate the use of a carboxylic acid-functionalized aldehyde (glyoxylic acid) to produce a diacid precursor, diglyoxylic acid xylose (DGAX), and the 
corresponding diester, dimethylglyoxylate xylose (DMGX), directly from both xylose and lignocellulosic biomass at high yield using scalable processes (Fig. 1). Polymerizing DMGX with a range of diols produced a family of polyesters, hereafter referred to as poly(alkylene xylosediglyoxylates) (PAX), that are bio-based, degradable, and chemically recyclable with combined high $\mathrm{T}_{\mathrm{g}}$, tough mechanical properties, strong gas barrier properties, and straightforward processability. 
A

\section{Xylose}

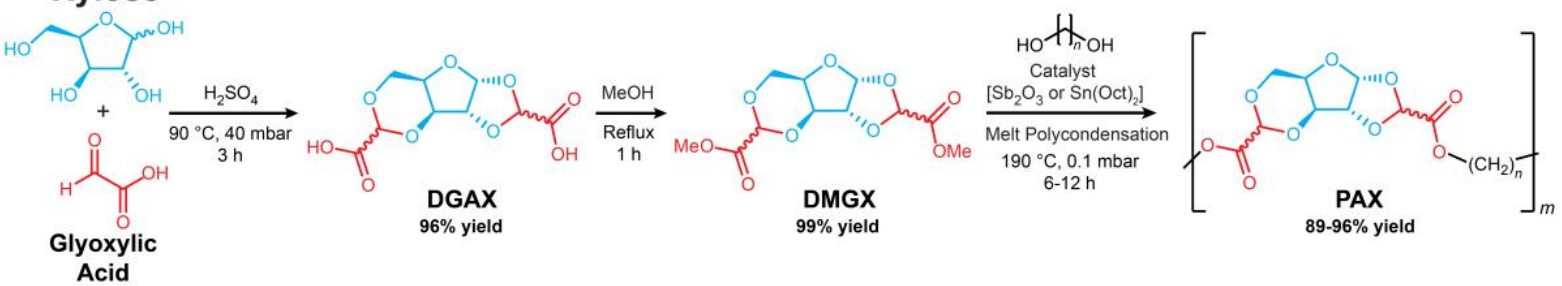

B

Lignocellulosic Biomass

Fractionated biomass

Subsequent upgrading

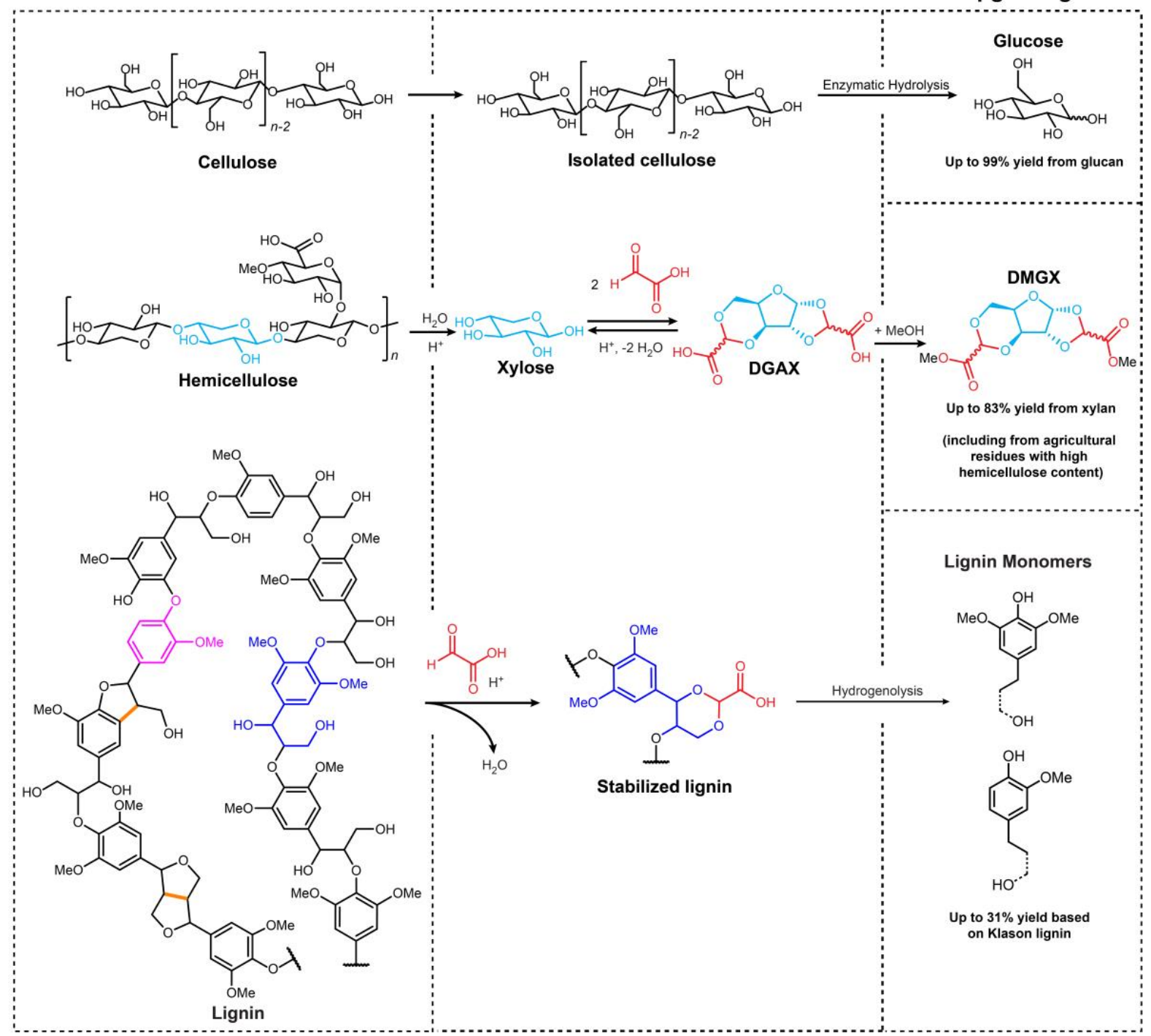

Fig. 1. Production of PAX polyesters directly from xylose or during integrated lignocellulosic biomass fractionation. (A) Direct production of DGAX from xylose, esterification to DMGX, and polymerization to poly(alkylene xylosediglyoxylate) (PAX). (B) Production of DMGX via aldehyde-assisted fractionation (AAF) of biomass followed by esterification with methanol. Both glyoxylic acid-stabilized lignin and cellulose-rich solids are also produced during fractionation and can be depolymerized to monomers. Glyoxylic acid prevents condensation of lignin and dehydration of xylose. Yields listed in the figure represent maximum product yields for each fraction, and not yields from a unique process (Fig. 2). As a benchmark for lignin yields (14), reductive catalytic fractionation of the same biomass resulted in a 56\% yield of lignin monomers based on Klason lignin (see SI section S1.7.9 for explanation of Klason lignin, and table S7 for direct hydrogenolysis data). 


\section{Results and Discussion}

We have developed a one-pot, two-stage process for producing DMGX from commercial xylose in $\geq 95 \%$ overall yield (supplementary information, or SI, section S1.7.1 and fig. S1). Xylose and an excess of glyoxylic acid are heated in a melt in the presence of sulfuric acid to yield DGAX as a mixture of 4 -stereoisomers (96\% yield) at a concentration of $54 \mathrm{wt} \%$ in the crude reaction mixture. DMGX is then produced by adding methanol under reflux (99\% yield with a crude concentration of $21 \mathrm{wt} \%$ ) and purified by distillation and recrystallization (figs. $\mathrm{S} 2$ and S3), or by direct precipitation from the reaction mixture (see SI section S1.7.5 and fig. S4). Single stereoisomers can also be partially chirally resolved by stepwise crystallization (see SI section S1.7.4).

To produce DMGX from lignocellulosic biomass, aldehyde-assisted fractionation (AAF) (11, 12) using glyoxylic acid is used to separate the biomass into its three principal components. Specifically, birch wood was treated with glyoxylic acid and a strong acid $\left(\mathrm{H}_{2} \mathrm{SO}_{4}\right.$ or $\left.\mathrm{HCl}\right)$, optionally in dioxane. After filtrating off the cellulose-rich solids and precipitating the glyoxylic acid-stabilized lignin (fig. S5), DMGX was synthesized and purified from the remaining liquor as described for commercial xylose (see SI section S1.7.6-S1.7.8 and fig. S6, A and B). Separately, the cellulose and lignin were depolymerized by enzymatic hydrolysis and hydrogenolysis, respectively, to determine the monomer yields that can be produced from these fractions (table S1-S2). We present three fractionation conditions: the first maximizes yields of all three components (Fig. 2A, with detailed mass balances in fig. S7), the second maximizes DMGX yields (Fig. 2B), and the third maximizes lignin monomers (Fig. 2C). These strategies illustrate potential trade-offs, but also opportunities to incorporate PAX production into an integrated biorefinery. The most favorable process would need to be selected for a particular biorefinery depending on local variables including location, feedstock, and market demand. Notably, producing DMGX directly from a biomass-derived liquor did not increase 
the number of separation steps required to achieve a polymerizable product. This is noteworthy because additional purification when using real plant-derived streams is often a major contributor to increased costs, and associated greenhouse gas emissions, of bio-derived products (15).

Though we used birch wood for the preliminary studies, certain abundant and inexpensive agricultural wastes with high hemicellulose and low lignin content (e.g., corn cobs, with $\sim 30 \%$ hemicellulose) (16), would be ideal feedstocks for low-cost DMGX production. As a demonstration, we were able to produce DMGX in a one pot process directly from raw corn cobs (not dried or pre-extracted) that contained $28 \mathrm{wt} \%$ hemicellulose (30 wt \% xylose when hydrolyzed) at $83 \%$ of theoretical yield (see SI section S1.7.17). This translates to $26 \mathrm{wt} \%$ of the non-edible waste biomass being directly incorporated into the DMGX plastic precursor. To our knowledge, this is the highest reported yield of a polymer precursor produced directly from lignocellulosic biomass. For comparison, and again, to our knowledge, synthesis of 5hydroxymethylfurfural (5-HMF), the precursor to FDCA, directly from biomass has only been achieved in a $\sim 50 \%$ of theoretical yield from lignocellulosic biomass and at product concentrations of around $1 \mathrm{wt} \%(17,18)$. Utilization of waste biomass could provide a low cost feedstock for DMGX production and could also ameliorate air quality and emissions issues in countries where crop waste is routinely burned, as it cannot be efficiently valorized (19).

When starting from either xylose or lignocellulosic biomass, the process is simple and scalable, uses an inexpensive mineral acid as the catalyst, and does not necessarily require a solvent. Glyoxylic acid is an inexpensive industrially available chemical $\left(\leq \$ 1 \cdot \mathrm{kg}^{-1}\right.$, see SI section S2.1.3), which can be produced renewably either by oxidation of bio-derived ethylene glycol or ethanol (20), or by electrochemical reduction of oxalic acid produced from $\mathrm{CO}_{2}(21,22)$. A technoeconomic analysis of the production of DMGX from commercial xylose (priced at 
$\$ 1 \cdot \mathrm{kg}^{-1}$ ) estimated a minimum DMGX selling price of $\$ 1577 \mathrm{USD} \cdot \mathrm{t}^{-1}$ (see SI section S2.1 and fig. S8) - a scenario that likely represents an upper bound of production costs, given that sugars from a biorefinery, or from agricultural residues, are projected to be significantly less expensive than current commercial xylose (i.e. $\left.<\$ 0.44 \cdot \mathrm{kg}^{-1}\right)(23)$. This price for DMGX is comparable to the market price of terephthalic acid (\$1445 USD $\left.t^{-1}\right)(24)$ and less than the average market price of $80 \%$ lactic acid $\left(\$ 1800 \mathrm{USD}^{-1} \mathrm{t}^{-1}\right)(15)$.

A cradle-to-gate life cycle analysis (LCA) of the simulated plant revealed a greenhouse warming potential (GWP) of $1.9 \mathrm{~kg} \mathrm{CO}_{2}$ equivalent per $\mathrm{kg}$ DMGX, which is $34 \%$ lower than the GWP of terephthalic acid, when using petroleum-based glyoxylic acid, xylose derived from viscose production (a common current source), and natural gas for steam and power production (see SI section S2.2 for a detailed description of the LCA and fig. S28). In this case, $84 \%$ of the calculated GWP of DMGX was associated with the production of glyoxylic acid. If glyoxylic acid was instead sourced from $\mathrm{CO}_{2}$, which may soon be feasible as its precursor, oxalic acid, is already being produced from captured $\mathrm{CO}_{2}$ at pilot scale (25), DMGX production was estimated to have $80 \%$ lower associated emissions as compared to terephthalic acid (GWP of $0.4 \mathrm{~kg} \mathrm{CO}_{2}$ equivalent per $\mathrm{kg}$ DMGX). This could be further reduced to $5 \%$ of terephthalic acid's GWP if agricultural residues were used for heat production as opposed to natural gas, which is typical in a biorefinery. 
A Glyoxylic Acid and Sulfuric Acid in Dioxane

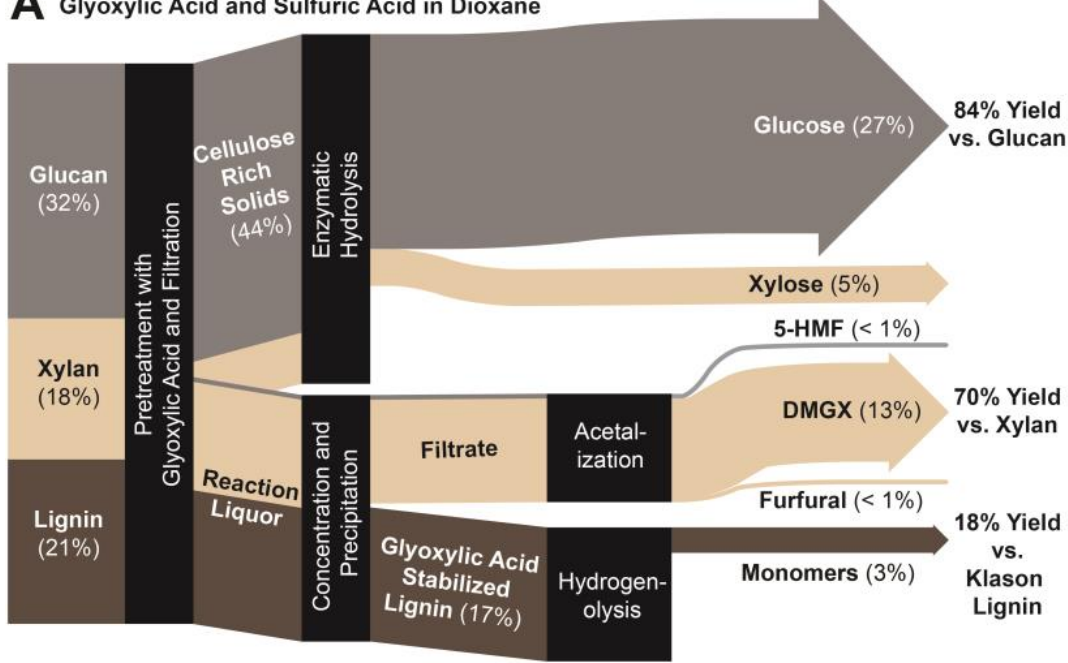

B Sulfuric Acid in Neat Glyoxylic Acid

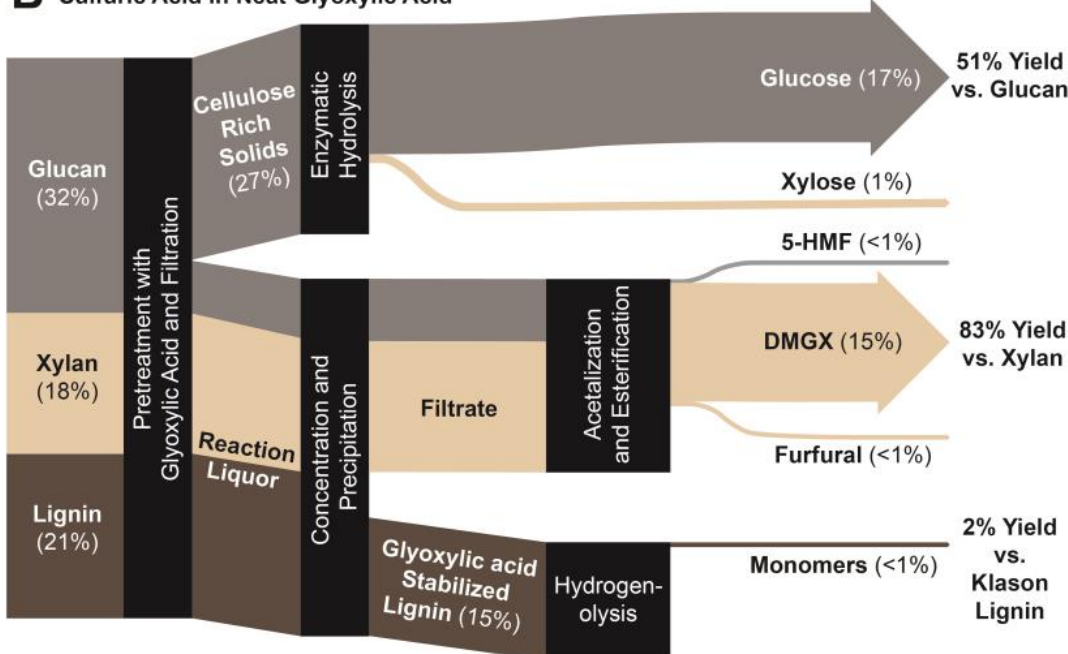

C Glyoxylic Acid and Hydrochloric Acid in Dioxane

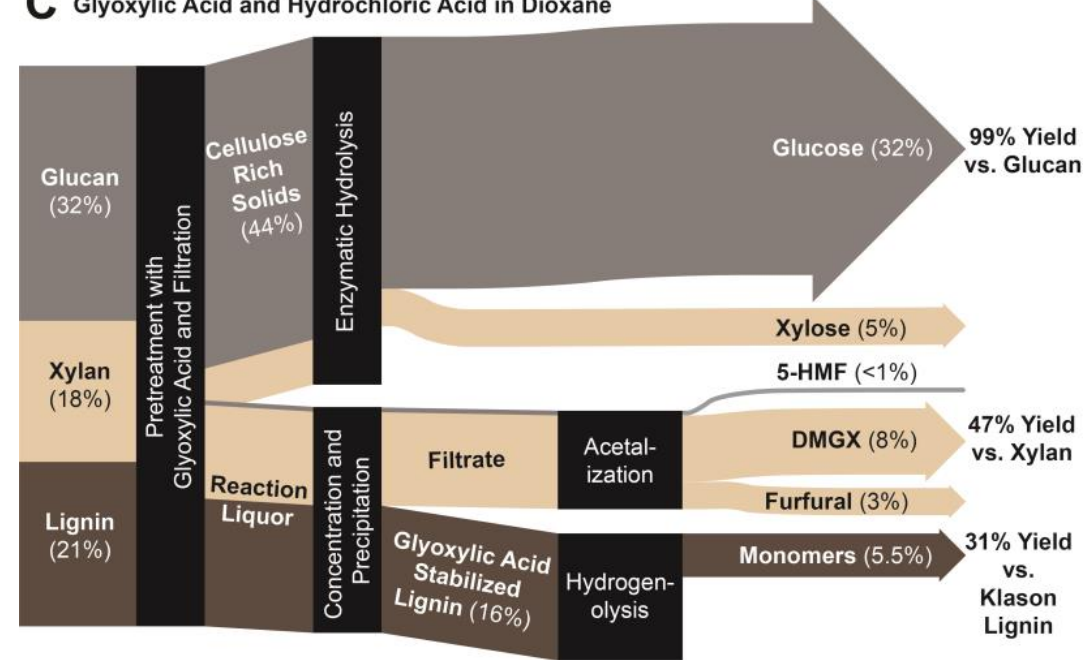

Fig. 2. Sankey diagram of the fractionation of birch wood with glyoxylic acid and the subsequent depolymerization and upgrading of the polysaccharide and lignin fractions. (A) Fractionation using sulfuric acid in dioxane (13 wt $\%$ birch wood, $7 \mathrm{wt} \%$ glyoxylic acid, $3 \mathrm{wt} \% \mathrm{H}_{2} \mathrm{SO}_{4}, 3 \mathrm{wt} \%$ water, and $74 \mathrm{wt} \%$ dioxane at $60{ }^{\circ} \mathrm{C}$ for $48 \mathrm{~h}$ ). (B) Fractionation using sulfuric acid in neat glyoxylic acid ( $13 \mathrm{wt} \%$ birch wood, $68 \mathrm{wt} \%$ glyoxylic acid, $2 \mathrm{wt} \% \mathrm{H}_{2} \mathrm{SO}_{4}, 17 \mathrm{wt} \%$ water at $110{ }^{\circ} \mathrm{C}$ for 2 h $15 \mathrm{~min}$ ). (C) Fractionation using hydrochloric acid in dioxane (13 wt\% birch wood, $7 \mathrm{wt} \%$ glyoxylic acid, $1 \mathrm{wt} \% \mathrm{HCl}, 4$ wt $\%$ water, $75 \mathrm{wt} \%$ dioxane at $60^{\circ} \mathrm{C}$ for $24 \mathrm{~h}$ ). The weight percentages of the sugars, DMGX, furfural, hydroxymethylfurfural (HMF), stabilized lignin, and lignin monomers have been corrected to match their constituent masses as part of the native biomass. The arrow widths are proportional to the corresponding molar yields. 
Xylose-derived DMGX (distilled 4-stereoisomer oil, see fig. S2 for full characterization) was polymerized with a range of aliphatic diols $\left(\mathrm{C}_{2}-\mathrm{C}_{6}\right)$ via melt condensation to produce amorphous polyesters $(89-96 \%$ yield). All polymerizations were conducted via transesterification of $>99 \%$ pure (by GC, SI section S.1.8.2) DMGX with excess diol and a Lewis acid catalyst with a final reaction temperature of $190-200{ }^{\circ} \mathrm{C}$ and 0.1 mbar of pressure. Reaction times slightly differed between diols but the decreased volatility of longer diols seemed to be compensated by the decrease in melt viscosity of the polymerization (increased mass transfer of the volatilized diol reaction byproduct). Likely due their high melt viscosities within the $190-200{ }^{\circ} \mathrm{C}$ temperature range, we were unable to obtain high molecular weight poly(ethylene xylosediglyoxylate) (PEX) and poly(propylene xylosediglyoxylate) (PPX). Both PEX and PPX were disperse, brittle, and had number average molecular weights $\left(\mathrm{M}_{\mathrm{n}}\right)$ of 4.2 $\mathrm{kDa}$ and $8.3 \mathrm{kDa}$, respectively (Fig. 3A). However, plastics synthesized from more flexible diols $\left(\mathrm{C}_{4}-\mathrm{C}_{6}\right)$ yielded polymers of $\mathrm{M}_{\mathrm{n}} \sim 45 \mathrm{kDa}$ with good dispersities ( 2.0) when using commercially relevant zinc acetate and antimony trioxide catalysts. Polymerization was also conducted with less toxic tin (II) 2-ethylhexanoate (TEH) catalyst to produce plastics with comparable properties and reaction times (see SI section S1.7.16). To explore the effect of molecular weight and stereochemistry on polymer properties, extra-high molecular weight poly(pentylene xylosediglyoxylate) (HMW-PPTX, $\mathrm{M}_{\mathrm{n}}=62 \mathrm{kDa}$ ) and single DMGX stereoisomer (see fig. S3 for complete characterization) poly(pentylene xylosediglyoxylate) (1S-PPTX, $\mathrm{M}_{\mathrm{n}}=39 \mathrm{kDa}$ ) were also synthesized. Matrix-assisted laser desorption/ionization time-of-flight mass spectroscopy (MALDI-TOF-MS, fig. S9) and 2D heteronuclear single quantum coherence spectroscopy (HSQC, fig. S10) confirmed that all of the intended polymer structures were obtained with no evidence of polymerization by-products or acetal rupture. 1SPPTX was also synthesized using DMGX produced from birch wood to confirm that true 
biomass-derived polymers could be synthesized without any substantial change in characteristics (see SI section S1.7.12 and fig. S6, C and D).

Polymer properties were unique compared to commercial bioplastics. Differential scanning calorimetry (DSC) and dynamic mechanical analysis (DMA, fig. S11A) revealed a range of glass transition temperatures $\left(\mathrm{T}_{\mathrm{g}}\right)$ from $72{ }^{\circ} \mathrm{C}$ for the poly(hexylene xylosediglyoxylate) (PHX) to $137{ }^{\circ} \mathrm{C}$ for PEX (Fig. 3B and table S3). The relatively high glass transition of these polyesters compared to commercial bioplastics and even PET $\left(80\right.$ to $85^{\circ} \mathrm{C}, 65$ to $75^{\circ} \mathrm{C}, 55$ to $65{ }^{\circ} \mathrm{C},-50$ to $-32{ }^{\circ} \mathrm{C}$, and $5{ }^{\circ} \mathrm{C}$ for poly(ethylene furanote) (PEF), PET, PLA, PBS, and poly(hydroxybutyrate) (PHB), respectively) is attributed to the rigid DMGX monomer. Thin cups made from PBX retain their mechanical strength when exposed to boiling water, whereas cups made from PLA or PET will become soft and lose their form (fig. S14H). Neither DSC nor X-ray diffraction (XRD) (fig. S12) showed evidence of crystalline phases in any of the DMGX polyesters, even for the polymer made from a single stereoisomer (1S-PPTX). The lack of crystallinity can be explained by the non-linear, non-planar, and asymmetric nature of DMGX (see the crystal structure of an isomer in fig. S3D). These thermal characteristics enabled the processing of the PHX, PPTX and poly(butylene xylosediglyoxylate) (PBX) polymers by common industrial techniques such as compression-molding (Fig. 3G), vacuumforming (Fig. 3H), injection-molding (Fig. 3I), or combined twin screw extrusion (fig. S14C) and 3D-printing (Fig. 3J) at temperatures as low as $140{ }^{\circ} \mathrm{C}$ and up to a maximum of $200{ }^{\circ} \mathrm{C}$ (for extrusion of PBX). These are well below the degradation onset temperatures $\left(319-344{ }^{\circ} \mathrm{C}\right)$ measured by thermogravimetric analysis (TGA) (fig. S11B). ${ }^{1} \mathrm{H}-\mathrm{NMR}$ and HSQC analysis of the PBX polymer before and after twin-screw extrusion and subsequent 3D printing (both at $200{ }^{\circ} \mathrm{C}$ ) revealed no evident change in chemical structure, or significant change in the number of ester end groups, indicating that PAX polymers can withstand high temperature, high shear 
processing without significant degradation or crosslinking (fig. S30). This stability under harsh processing conditions could facilitate mechanical recycling of the polymer.

Tensile testing on compression-molded dog bones (table S3 and fig. S11D) revealed that PHX and PPTX had high ultimate tensile strength and ductility - comparable to fossil-based bisphenol A polycarbonate and PET (Fig. 3C). PBX showed greater strength, but significantly reduced flexibility, making it more comparable to PEF and PLA. Both PEX and PPX were too brittle for mechanical analysis. The 1S-PPTX polymer had slightly improved tensile strength and rheological properties (fig. S13) compared to PPTX, even at lower $\mathrm{M}_{\mathrm{n}}$ (39 kDa vs. 50 $\mathrm{kDa}$ ). This improvement was likely due to a more ordered orientation of polymer chains. However, other than increased melt viscosities, the mixed stereochemistry of the 4-isomer PAX polymers, for the most part, did not result in inferior material properties that would hinder their commercialization. Tensile testing of 1S-PPTX using dog bones prepared via injection molding (without extruder screws) to avoid defects seen in the compression molded samples (e.g., air bubbles, fig. S14E), revealed a significantly higher ultimate tensile strength. Therefore, tensile strengths reported in this study may be underestimated and should be interpreted with caution.

The measured oxygen transmission rates (OTR) of films of PHX, PPTX, and PBX were similar to those of PET (26) with slightly higher water vapor transmission rates (WVTR) (Fig. 3D and table S3) - potentially lending themselves to food packaging applications. The low oxygen permeability of these amorphous polymers as compared to semi-crystalline, degradable commercial bioplastics (PLA, PHB, PBS) likely results from the polar and barrier-like nature of the rigid DMGX monomer which likely leads to decreased oxygen solubility and diffusion, respectively. The slightly higher WVTRs, as compared to PET, likely result from the hydrophilic nature of the DMGX monomer (greater water solubility), but they still demonstrate $<50 \%$ of the water permeability of PLA. Current commercial bioplastics - other than 
emerging options such as poly(glycolic acid) (PGA), a bioplastic for which there is a lack of inexpensive production routes (27); or PEF, which is non-biodegradable and involves a much more complex production route from biomass - have had trouble addressing food packaging requirements due to their generally poor gas barrier properties or poor ductility (28).

PAX polymers derived from the distilled 4-stereoisomer DMGX oil were yellow in color (fig. S15), which is undesirable in many applications. Increasing the purity of the DMGX monomer by recrystallization resulted in plastics that could be processed into fibers and films with significantly reduced coloration (Fig. 3G and fig. S14A and B). Notably, the 4S-DMGX oil could be directly recrystallized as a mixture of 4 isomers to remove colored impurities, or the most abundant isomer could be first selectively crystallized out of the mixture, followed by crystallization of the remaining isomers, using stepwise temperature recrystallization (see SI section S1.7.5). In thicker PAX samples, however, even the crystallized DMGX resulted in slight yellow coloration when zinc acetate or dibutyltin oxide (DBTO) were used as catalysts (Figure 3I). Fortunately, initial tests indicate that optimized catalyst and antioxidant combinations can be used to dramatically reduce discoloring side reactions during polycondensation while maintaining similar reaction times (see SI section S1.7.16). Tin (II) 2ethylhexanoate (TEH, $0.008 \mathrm{~mol} \%$ ) and triphenyl phosphite (TTP, $0.17 \mathrm{~mol} \%$ ) were used to produce near-colorless and transparent PHX products (Fig. 3E, F, and H). DMA analysis of the polymers produced using TEH reveal essentially identical thermomechanical properties, except for a slightly superior glass transition by onset of storage modulus decay, as those polymerized with the DBTO catalyst (fig. S27). 
A

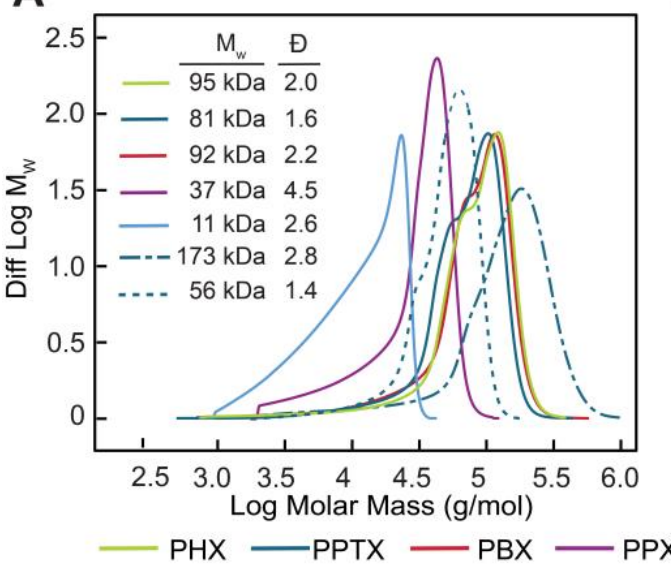

C

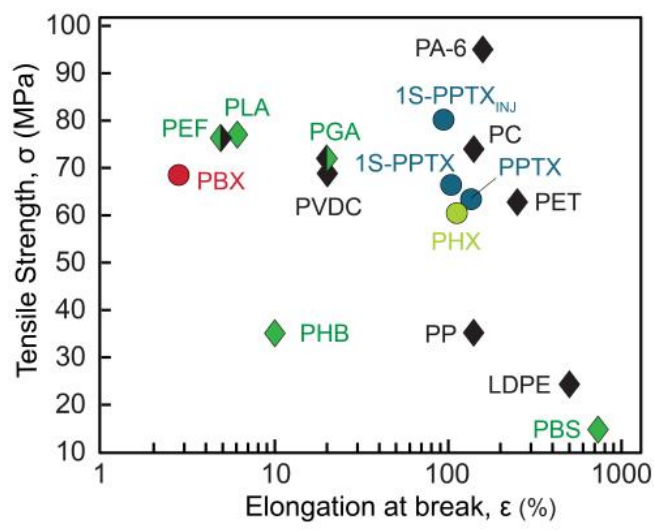

E

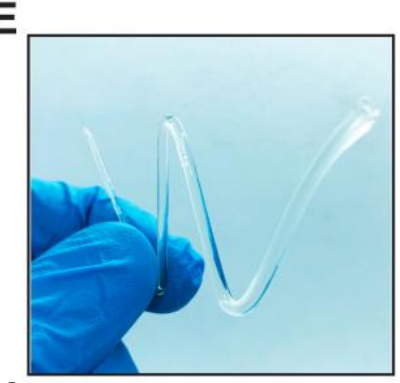

H

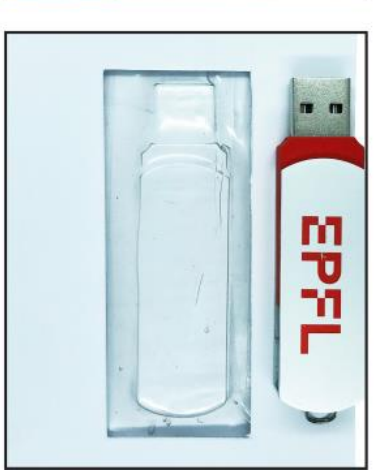

$\mathbf{F}$
B

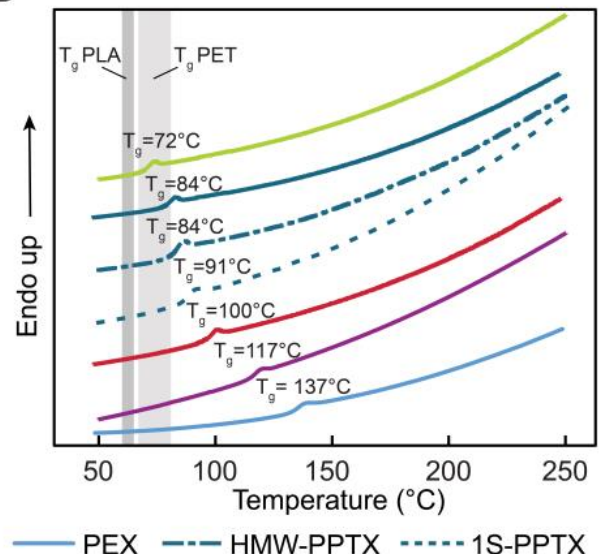

D

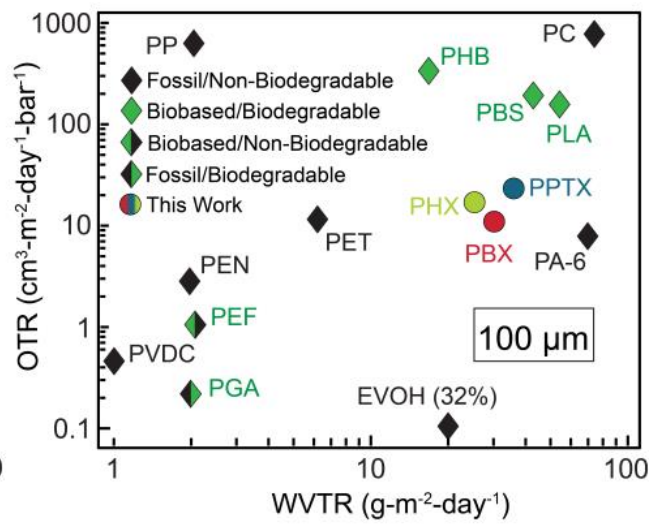

G
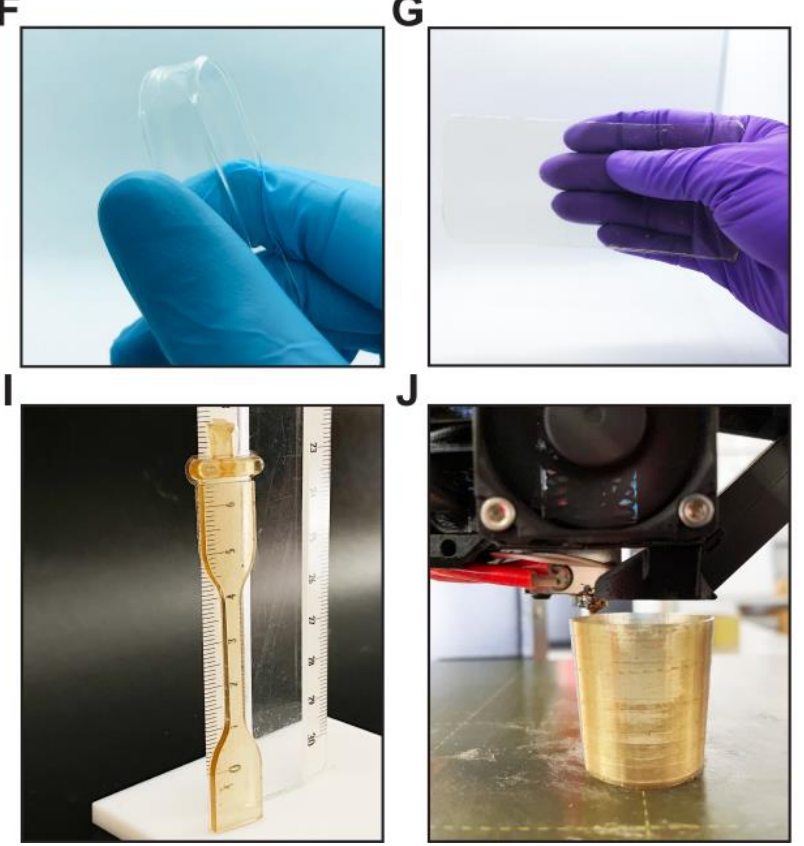

Fig. 3. Properties of PAX polymers. (A) Molecular weight distributions. (B) DSC curves. (C) Elongation at break vs. ultimate tensile strength for PAX and other commercial plastics (see table S4 for numerical values and references). (D) WVTR $\left(37.8{ }^{\circ} \mathrm{C}, 90 \%\right.$ relative humidity, $\left.100 \mu \mathrm{m}\right)$ vs. OTR $\left(23{ }^{\circ} \mathrm{C}, 50 \%\right.$ relative humidity, 1 bar, $\left.100 \mu \mathrm{m}\right)$ for PAX and other commercial plastics (see table S5 for numerical values and references). (E) Fiber pulled quickly, and (F) slowly, from molten 1S-PPTX using optimized catalyst (TEH) and antioxidant (TTP) combination. (G) Compression-molded film of 1S-PPTX using DBTO as catalyst with no antioxidant with $500 \mu \mathrm{m}$ thickness. (H) Packaging for USB key produced by vacuum-forming of PHX (500 $\mu \mathrm{m}$ thickness) produced with optimized catalyst and antioxidant. (I) Injection molded dog bone using 1S-PPTX polymer using DBTO as catalyst with no antioxidant. Ruler graduations are in centimeters. (J) 3D-printed cup using twinscrew extruded PBX filament. 
Chemical recycling of PAX from a mixed plastic waste-stream was demonstrated by alcoholysis (Fig. 4A-C and SI section S1.7.13). Compression-molded PHX was fully depolymerized and solvated from a mix of common plastics (polyamide, polyethylene, polypropylene, polycarbonate, and PET) with a $96 \%$ recovery of DMGX by refluxing in acidic methanol ( $\left.25 \mathrm{mM} \mathrm{H}_{2} \mathrm{SO}_{4}\right)$ for $5 \mathrm{~h}$, without any evident contaminants from the non-PAX plastics (fig. S16). After filtering off the residual plastics (fig. S14D), the DMGX and 1,6-hexanediol were separated by liquid-liquid extraction and distilled to produce pure monomers in $82 \%$ and $74 \%$ yields, respectively (see fig. S17, A and B for 2D-NMRs of the recycled monomers). The reduced yields of purified DMGX result from separation losses at small scale in batch, and would likely be substantially reduced in a large-scale, optimized, continuous process. PHX was then re-synthesized using the recycled monomers. HSQC (fig. S17, C and D) and DMA (Fig. 4C) revealed no significant change in chemical structure or thermomechanical properties between the virgin and recycled polymers. The slightly lower $\mathrm{T}_{\mathrm{g}}$ of the virgin PHX was attributed to the removal of a polymerization antioxidant (Irganox 1010) during the chemical recycling process, demonstrating that the presence of polymerization additives will likely not hinder recyclability. Antioxidants were added to prevent small amounts of oxidation side reactions that can often cause a yellow tint in the final polyesters (this is typically done in industry). Low temperature methanolysis from a mixed plastic stream at atmospheric pressure using standard separation techniques (extraction and distillation or crystallization) may make industrial chemical recycling of PAX more economically feasible compared to the high temperature and pressure operations required for petroleum-based plastics such as PET (29). 

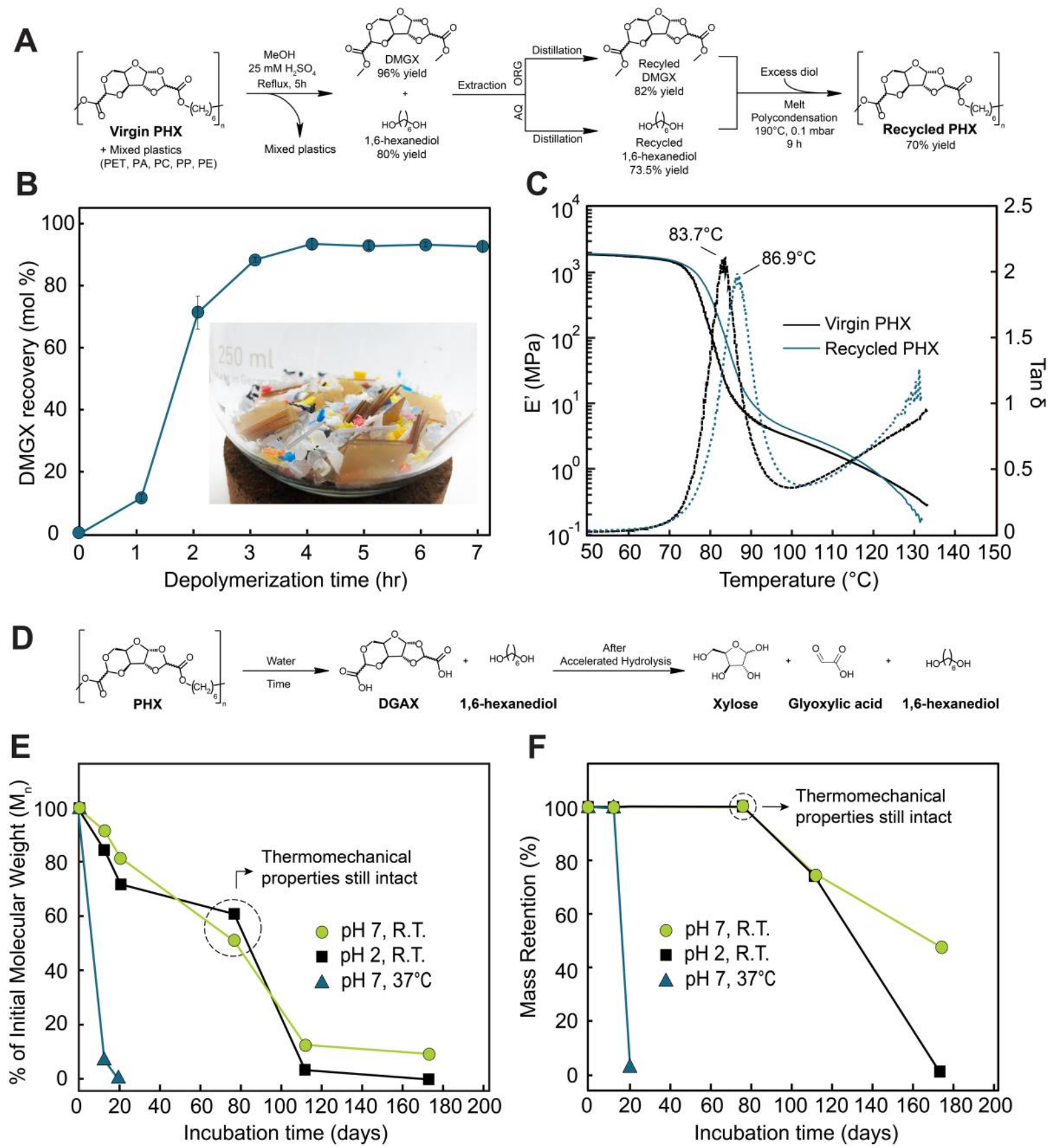

Fig. 4. Chemical recycling and hydrolytic stability studies. (A) Process schematic for chemical recycling of PHX. Yield of the recycled plastic is based on DMGX utilization. (B) DMGX recovery upon depolymerization of compression-molded PHX via alcoholysis in methanol under reflux with $25 \mathrm{mM}$ sulfuric acid. Error bars are standard errors $(\mathrm{n}=3)$. Image shows the simulated mixed plastic waste stream used for chemical recycling demonstration. (C) DMA curves of virgin and chemically recycled PHX. Tan delta curves are dashed lines. (D) Schematic of PHX hydrolysis. PHX hydrolyzed to DGAX and diol monomers in the extended hydrolytic studies at room temperature and $37{ }^{\circ} \mathrm{C}$. Further hydrolysis of DGAX to xylose and glyoxylic acid occurred in the accelerated hydrolysis conditions (water at $100{ }^{\circ} \mathbf{C}$ ). $(\mathbf{E})$ Percent of initial number average molecular weight as a function of incubation time of compression-molded PHX samples (initial $\mathrm{M}_{\mathrm{n}}$ of $43 \mathrm{kDa}$ ) immersed in buffered solutions at the indicated $\mathrm{pH}$ and temperature (R.T.= room temperature). (F) Mass retention of the plastic samples measured after removal from buffered solutions, and thorough washing with DI water and drying. Solid lines were added to guide the eye and do not represent a model. 
As only $9 \%$ of plastic is currently recycled and $79 \%$ ends up in landfills or the environment (1), the degradation of plastics in aqueous environments is an important feature of a material's sustainability (30). In this context, hydrolytic stability tests were performed on compressionmolded samples of PHX (fig. S15D) with an initial $M_{n}$ of $43 \mathrm{kDa}$ over the course of 180 days under neutral and acidic conditions at room temperature, and under neutral conditions at $37{ }^{\circ} \mathrm{C}$ (Fig. 4D-F). After 77 days at room temperature for both the $\mathrm{pH} 2$ and $\mathrm{pH} 7$ solutions, the $\mathrm{M}_{\mathrm{n}}$ declined to $\sim 23 \mathrm{kDa}\left(\sim 55 \%\right.$ of the initial $\left.\mathrm{M}_{\mathrm{n}}\right)$, with no mass loss. DMA analysis of these degraded samples revealed no significant change in thermomechanical properties compared to the original polymer, other than a slightly reduced melt strength (fig. S18). Following the 77day timepoint, the plastics continued to hydrolyze and began to dissolve in the aqueous solutions. After 173 days in the $\mathrm{pH} 2$ water, the plastic was essentially fully dissolved $(<2 \%$ of initial mass remaining), but in the $\mathrm{pH} 7$ water, $47 \mathrm{wt} / \mathrm{wt} \%$ of the plastic remained with a reduced $\mathrm{M}_{\mathrm{n}}$ of $4 \mathrm{kDa}\left(9 \%\right.$ of initial $\mathrm{M}_{\mathrm{n}}$ ). When the hydrolytic stability tests were performed at an elevated temperature of $37{ }^{\circ} \mathrm{C}$ at $\mathrm{pH} 7$, the $\mathrm{M}_{\mathrm{n}}$ declined to $3 \mathrm{kDa}\left(7 \%\right.$ of initial $\mathrm{M}_{\mathrm{n}}$ ) with no mass loss after 13 days, while after 20 days, the plastic was essentially fully dissolved $(<4 \%$ of initial mass). The degraded plastic dissolved to a mixture of monomers (DGAX and 1,6hexanediol) and oligomers (as determined by HPLC analysis, fig. S31).

Hydrolytic stability tests of the PHX, PBX, and 1S-PHX polymers with identical starting molecular weights $\left(\mathrm{M}_{\mathrm{n}} \sim 20 \mathrm{kDa}\right)$ at these conditions $\left(\mathrm{pH} 7,37^{\circ} \mathrm{C}\right)$ revealed that the $\mathrm{PBX}$ and 1S-PHX polymers demonstrated a $30 \%$ and $90 \%$ increase in hydrolytic stability (incubation time with $>99 \%$ mass retention) as compared to the PHX polymer, respectively (fig. S29). This indicates that the single isomer polymers could likely be used for products that require a longer shelf-life in humid environments. For applications in which hydrolytic cleavage rates need to be even further reduced, strategies including the use of anti-hydrolysis agents (e.g., aromatic carbodiimides) have already been demonstrated to significantly increase the 
hydrolytic stability of degradable PLA and PGA, and could presumably be applied to PAX $(31,32)$.

To gain insight into the long-term fate of the resulting DGAX in water, accelerated aqueous depolymerization of PAX by boiling compression-molded PHX in deuterium oxide $\left(\mathrm{D}_{2} \mathrm{O}\right)$ revealed depolymerization to diacid (DGAX) and 1,6-hexanediol after $9 \mathrm{~h}$ and subsequent partial hydrolysis of DGAX to glyoxylic acid and xylose beginning after $17 \mathrm{~h}$ and continuing throughout the $120 \mathrm{~h}$ experiment (see SI section S1.7.15 and figs. S19-S21). Though standardized biodegradation and composting tests still need to be performed, these initial results suggest that the long-term environmental fate of the polymer in the presence of water is likely to be hydrolysis to xylose, glyoxylic acid, and diol - all of which are non-toxic and biodegradable (33-36). This potential fate demonstrates that the preservation of the largely unmodified carbohydrate core, in addition to greatly facilitating the polyester's production and ensuring high atom economy, leads to a natural degradation pathway back to sugars.

\section{Materials and Methods}

The methods for DMGX synthesis both from xylose and lignocellulosic biomass, as well as their subsequent polymerizations to produce polyesters, are briefly described in the main text. More detailed synthesis methods, material description and detailed analytical and material characterization procedures are given in the supplementary information (SI).

\section{References}

1. R. Geyer, J. R. Jambeck, K. L. Law, Production, use, and fate of all plastics ever made. Sci. Adv. 3, e1700782 (2017).

2. M. A. Hillmyer, The promise of plastics from plants. Science. 358, 868-870 (2017).

3. Y. Zhu, C. Romain, C. K. Williams, Sustainable polymers from renewable resources. Nature. 540, 354-362 (2016).

4. G. Wypych, Handbook of Polymers (Elsevier, 2016).

5. M. Rose, R. Palkovits, Isosorbide as a Renewable Platform chemical for Versatile Applications-Quo Vadis? ChemSusChem. 5, 167-176 (2012). 
6. S. Muñoz-Guerra, C. Lavilla, C. Japu, A. Martínez de Ilarduya, Renewable terephthalate polyesters from carbohydrate-based bicyclic monomers. Green Chem. 16, 1716-1739 (2014).

7. T. Shahzadi, S. Mehmood, M. Irshad, Z. Anwar, A. Afroz, N. Zeeshan, U. Rashid, K. Sughra, Advances in lignocellulosic biotechnology: A brief review on lignocellulosic biomass and cellulases. ABB. 05, 246-251 (2014).

8. S. N. Naik, V. V. Goud, P. K. Rout, A. K. Dalai, Production of first and second generation biofuels: A comprehensive review. Renewable and Sustainable Energy Reviews. 14, 578-597 (2010).

9. M. Häußler, M. Eck, D. Rothauer, S. Mecking, Closed-loop recycling of polyethylenelike materials. Nature. 590, 423-427 (2021).

10. B. M. Upton, A. M. Kasko, Strategies for the Conversion of Lignin to High-Value Polymeric Materials: Review and Perspective. Chemical Reviews. 116, 2275-2306 (2016).

11. L. Shuai, M. T. Amiri, Y. M. Questell-Santiago, F. Héroguel, Y. Li, H. Kim, R. Meilan, C. Chapple, J. Ralph, J. S. Luterbacher, Formaldehyde stabilization facilitates lignin monomer production during biomass depolymerization. Science. 354, 329-333 (2016).

12. W. Lan, M. T. Amiri, C. M. Hunston, J. S. Luterbacher, Protection Group Effects During $\alpha, \gamma$-Diol Lignin Stabilization Promote High-Selectivity Monomer Production. Angewandte Chemie International Edition. 57, 1356-1360 (2018).

13. Y. M. Questell-Santiago, R. Zambrano-Varela, M. Talebi Amiri, J. S. Luterbacher, Carbohydrate stabilization extends the kinetic limits of chemical polysaccharide depolymerization. Nature Chemistry. 10, 1222-1228 (2018).

14. M. Talebi Amiri, G. R. Dick, Y. M. Questell-Santiago, J. S. Luterbacher, Fractionation of lignocellulosic biomass to produce uncondensed aldehyde-stabilized lignin. Nat Protoc. 14, 921-954 (2019).

15. M. J. Biddy, C. Scarlata, C. Kinchin, "Chemicals from Biomass: A Market Assessment of Bioproducts with Near-Term Potential” (NREL/TP--5100-65509, 1244312, 2016), p. NREL/TP--5100-65509, 1244312, doi:10.2172/1244312.

16. M. Pointner, P. Kuttner, T. Obrlik, Composition of corncobs as a substrate for fermentation of biofuels. Agronomy Research. 12, 391-396 (2014).

17. J. S. Luterbacher, D. M. Alonso, J. A. Dumesic, Targeted chemical upgrading of lignocellulosic biomass to platform molecules. Green Chemistry. 16, 4816-4838 (2014). 
18. I. K. M. Yu, D. C. W. Tsang, Conversion of biomass to hydroxymethylfurfural: A review of catalytic systems and underlying mechanisms. Bioresource Technology. 238, 716-732 (2017).

19. M.-T. Cheng, C.-L. Horng, Y.-R. Su, L.-K. Lin, Y.-C. Lin, C. C.-K. Chou, Particulate matter characteristics during agricultural waste burning in Taichung City, Taiwan. Journal of Hazardous Materials. 165, 187-192 (2009).

20. I. Van Nieuwenhove, T. Renders, J. Lauwaert, T. De Roo, J. De Clercq, A. Verberckmoes, Biobased Resins Using Lignin and Glyoxal. ACS Sustainable Chem. Eng. 8, 18789-18809 (2020).

21. D. J. Pickett, K. S. Yap, A study of the production of glyoxylic acid by the electrochemical reduction of oxalic acid solutions. J Appl Electrochem. 4, 17-23 (1974).

22. B. Scharbert, P. Babusiaux, Electrochemical process for reducing oxalic acid to glyoxylic acid (1995), (available at https://patents.google.com/patent/US5395488A/en).

23. R. Davis, L. Tao, C. Scarlata, E. C. D. Tan, J. Ross, J. Lukas, D. Sexton, "Process Design and Economics for the Conversion of Lignocellulosic Biomass to Hydrocarbons: Dilute-Acid and Enzymatic Deconstruction of Biomass to Sugars and Catalytic Conversion of Sugars to Hydrocarbons" (NREL/TP--5100-62498, 1176746, 2015), p. NREL/TP--5100-62498, 1176746, , doi:10.2172/1176746.

24. Purified Terephthalic Acid (PTA) Prices and Pricing Information. ICIS Explore, (available at https://www.icis.com/explore/resources/news/2007/11/06/9076460/purifiedterephthalic-acid-pta-prices-and-pricing-information).

25. E. Schuler, M. Demetriou, N. R. Shiju, G.-J. M. Gruter, Towards Sustainable Oxalic Acid from CO2 and Biomass. ChemSusChem. n/a, doi:10.1002/cssc.202101272.

26. J. Lange, Y. Wyser, Recent innovations in barrier technologies for plastic packaginga review. Packaging Technology and Science. 16, 149-158 (2003).

27. K. Yamane, H. Sato, Y. Ichikawa, K. Sunagawa, Y. Shigaki, Development of an industrial 775 (2014).

28. X. Zhao, K. Cornish, Y. Vodovotz, Narrowing the Gap for Bioplastic Use in Food Packaging: An Update. Environ. Sci. Technol. 54, 4712-4732 (2020). 
29. N. George, T. Kurian, Recent Developments in the Chemical Recycling of Postconsumer Poly(ethylene terephthalate) Waste. Ind. Eng. Chem. Res. 53, 1418514198 (2014).

30. R. A. Gross, B. Kalra, Biodegradable Polymers for the Environment. Science. 297, 803-807 (2002).

31. P. Stloukal, G. Jandikova, M. Koutny, V. Sedlařík, Carbodiimide additive to control hydrolytic stability and biodegradability of PLA. Polymer Testing. 54, 19-28 (2016).

32. H. Sato, F. Akutsu, F. Kobayashi, Y. Okada, Process for producing aliphatic polyester (2009), (available at https://patents.google.com/patent/US7538178B2/en).

33. Glyoxylic acid - Registration Dossier - ECHA, (available at https://echa.europa.eu/registration-dossier/-/registered-dossier/14954/6/2/8).

34. Butane-1,4-diol - Registration Dossier - ECHA, (available at https://echa.europa.eu/registration-dossier/-/registered-dossier/15496/1).

35. Pentane-1,5-diol - Registration Dossier - ECHA, (available at https://echa.europa.eu/registration-dossier/-/registered-dossier/14818/6/2/8).

36. Hexane-1,6-diol - Registration Dossier - ECHA, (available at https://echa.europa.eu/registration-dossier/-/registered-dossier/15109/6/2/1).

37. Sluiter, J. \& Sluiter, A., "Summative Mass Closure: Laboratory Analytical Procedure (LAP) Review and Integration” (National Renewable Energy Laboratory, 2011), (available at https://www.nrel.gov/docs/gen/fy11/48087.pdf).

38. B. Adney, J. Baker, Measurement of Cellulase Activities: Laboratory Analytical Procedure (LAP); Issue Date: 08/12/1996. Technical Report, 11 (2008).

39. M. M. Abu-Omar, K. Barta, G. T. Beckham, J. S. Luterbacher, J. Ralph, R. Rinaldi, Y. Román-Leshkov, J. S. M. Samec, B. F. Sels, F. Wang, Guidelines for performing lignin-first biorefining. Energy Environ. Sci. 14, 262-292 (2021).

40. G. M. Sheldrick, SHELXT - Integrated space-group and crystal-structure determination. Acta Crystallogr A Found Adv. 71, 3-8 (2015).

41. O. V. Dolomanov, L. J. Bourhis, R. J. Gildea, J. A. K. Howard, H. Puschmann, OLEX2 : a complete structure solution, refinement and analysis program. J Appl Crystallogr. 42, 339-341 (2009).

42. G. M. Sheldrick, Crystal structure refinement with SHELXL. Acta Crystallogr C Struct Chem. 71, 3-8 (2015).

43. R. Davis, L. Tao, E. C. D. Tan, M. J. Biddy, G. T. Beckham, C. Scarlata, J. Jacobson, K. Cafferty, J. Ross, J. Lukas, D. Knorr, P. Schoen, "Process Design and Economics 
for the Conversion of Lignocellulosic Biomass to Hydrocarbons: Dilute-Acid and Enzymatic Deconstruction of Biomass to Sugars and Biological Conversion of Sugars to Hydrocarbons" (NREL/TP-5100-60223, 1107470, 2013), p. NREL/TP-5100-60223, 1107470, doi:10.2172/1107470.

44. N. R. Trivedi, S. B. Chandalia, Synthesis of Alkyl Glyoxylates. Organic Process Research \& Development. 2, 332-333 (1998).

45. A. Sajitos, Process for the preparation of glyoxylic acid and glyoxylic acid derivatives. (1991).

46. Top Quality Glyoxylic Acid Monohydrate 98.5\% With Fast Delivery. www.alibaba.com, (available at //www.alibaba.com/product-detail/Top-qualityGlyoxylic-acid-monohydrate-98_62422265476.html).

47. Glyoxylic Acid Cas 298-12-4 - Buy Glyoxylic Acid, www.alibaba.com, (available at https://www.alibaba.com/product-detail/Glyoxylic-acid-CAS-298-12-

4_1600080630437.html?spm=a2700.galleryofferlist.normal_offer.d_image.2a9c74f5 $5 \mathrm{hRebc})$.

48. A. H. Motagamwala, W. Won, C. Sener, D. M. Alonso, C. T. Maravelias, J. A. Dumesic, Toward biomass-derived renewable plastics: Production of 2,5furandicarboxylic acid from fructose. Sci. Adv. 4, eaap9722 (2018).

49. L. Shen, E. Worrell, M. K. Patel, Environmental impact assessment of man-made cellulose fibres. Resources, Conservation and Recycling. 55, 260-274 (2010).

50. European Commission Decision 2007/589/EC: Official Journal of the European Commission, 31.8.2007, L229/1.

51. M. Li, T. Ruddy, D. Fahey, D. H. Busch, B. Subramaniam, Terephthalic Acid Production via Greener Spray Process: Comparative Economic and Environmental Impact Assessments with Mid-Century Process. ACS Sustainable Chem. Eng. 2, 823835 (2014).

52. E. Schuler, M. Demetriou, N. R. Shiju, G.-J. M. Gruter, Towards Sustainable Oxalic Acid from CO2 and Biomass. ChemSusChem. n/a, doi:10.1002/cssc.202101272.

53. H. H. Khoo, I. Halim, A. D. Handoko, LCA of electrochemical reduction of CO2 to ethylene. Journal of CO2 Utilization. 41, 101229 (2020).

54. European Commission. Joint Research Centre., Solid and gaseous bioenergy pathways: input values and GHG emissions: calculated according to the methodology set in COM(2016) 767. (Publications Office, LU, 2017; https://data.europa.eu/doi/10.2790/27486). 
55. A. El-Hadi, R. Schnabel, E. Straube, G. Müller, S. Henning, Correlation between degree of crystallinity, morphology, glass temperature, mechanical properties and biodegradation of poly (3-hydroxyalkanoate) PHAs and their blends. Polymer Testing. 21, 665-674 (2002).

56. V. Jost, Packaging related properties of commercially available biopolymers - An overview of the status quo. Express Polym. Lett. 12, 429-435 (2018).

57. J.-G. Rosenboom, D. K. Hohl, P. Fleckenstein, G. Storti, M. Morbidelli, Bottle-grade polyethylene furanoate from ring-opening polymerisation of cyclic oligomers. Nat Commun. 9, 2701 (2018).

58. G. L. Robertson, in Encyclopedia of Agriculture and Food Systems, N. K. Van Alfen, Ed. (Academic Press, Oxford, 2014; https://www.sciencedirect.com/science/article/pii/B9780444525123000632), pp. 232249.

59. M. Schmid, K. Dallmann, E. Bugnicourt, D. Cordoni, F. Wild, A. Lazzeri, K. Noller, Properties of Whey-Protein-Coated Films and Laminates as Novel Recyclable Food Packaging Materials with Excellent Barrier Properties. International Journal of Polymer Science. 2012, 1-7 (2012).

60. J. Xu, P. H. Manepalli, L. Zhu, S. Narayan-Sarathy, S. Alavi, Morphological, barrier and mechanical properties of films from poly (butylene succinate) reinforced with nanocrystalline cellulose and chitin whiskers using melt extrusion. J Polym Res. 26, 188 (2019).

61. Polyethylene Furanoate (PEF) Material Guide: Bio-based Polymer, (available at https://omnexus.specialchem.com/selection-guide/polyethylene-furanoate-pefbioplastic).

62. Kuredux Polyglycolic Acid (PGA) Resin, (available at http://www.kureha.com/pdfs/Kuredux_brochure.pdf).

63. O. Akin, F. Tihminlioglu, Effects of Organo-Modified Clay Addition and Temperature on the Water Vapor Barrier Properties of Polyhydroxy Butyrate Homo and Copolymer Nanocomposite Films for Packaging Applications. J Polym Environ. 26, 1121-1132 (2018).

64. C. T. Prontera, G. Sico, M. Montanino, A. De Girolamo Del Mauro, P. Tassini, M. G. Maglione, C. Minarini, P. Manini, Sustainable, Fluorine-Free, Low Cost and Easily Processable Materials for Hydrophobic Coatings on Flexible Plastic Substrates. Materials. 12, 2234 (2019). 
65. BLS Series Report: U.S. Bureau of Labor Statistics, (available at https://data.bls.gov/cgi-bin/srgate).

66. Hot Sell Food Grade 99\% Min D Xylose Price - Buy D Xylose Price Product on Alibaba.com, (available at https://www.alibaba.com/product-detail/Hot-sell-foodgrade-99min_62132025664.html?spm=a2700.7724857.normalList.220.6e7f64aeuIwDEg).

67. Pricing | Methanex Corporation, (available at https://www.methanex.com/ourbusiness/pricing).

68. J. S. Luterbacher, J. M. Rand, D. M. Alonso, J. Han, J. T. Youngquist, C. T. Maravelias, B. F. Pfleger, J. A. Dumesic, Nonenzymatic Sugar Production from Biomass Using Biomass-Derived -Valerolactone. Science. 343, 277-280 (2014).

69. T. Ivanović, R. Hischier, C. Som, Bio-Based Polyester Fiber Substitutes: From GWP to a More Comprehensive Environmental Analysis. Applied Sciences. 11, 2993 (2021).

70. Goodridge, F., et al. "Scale-up Studies of the Electrolytic Reduction of Oxalic to Glyoxylic Acid.” Journal of Applied Electrochemistry, vol. 10, no. 1, Jan. 1980, pp. 55-60, doi:10.1007/BF00937338.

Acknowledgments: The authors thank Michael Studer from the Bern University of Applied Sciences (Switzerland) for providing various wood samples, IP-Suisse for providing corn cob samples, Chloé Wegmann for compositional analyses of the biomass feedstocks, Mélanie Hannebelle and Antoine Gagliardi for assisting with the vacuum forming, Farzaneh Fadaei Tirani for crystal structure determination, Stefania Bertella for assistance with graphic design and illustrations, Mariella S. Vieli for assistance with DSC measurements, and Arnaud Magrez for powder XRD measurements.

\section{Funding:}

Swiss National Science Foundation grant (CRSII5_180258, JSL, LPM)

European Research Council (ERC) under the European Union's Horizon 2020 research and innovation program (Starting grant: CATACOAT, No. 758653, JSL, GRD)

Swiss Competence Center for Energy Research: Biomass for a Swiss Energy Future through the Swiss Commission for Technology and Innovation grant (KTI.2014.0116, JSL)

EPFL

Author contributions: LPM and JSL conceived of the project and designed the research. LPM performed most of the experiments and drafting of the manuscript. GRD performed most of the biomass fractionation and lignin upgrading experiments and helped design syntheses. AD performed most of the material characterization experiments and was supervised by YL and VM. MAH, with the assistance of CR, performed the catalyst optimization and some of the polymerization, purification and material characterization experiments. MJJ performed the life 
cycle analysis supervised by FM. TR performed the pretreatment experiments on the corn cob feedstock. IS performed GPC experiments supervised by AP. HAK helped design some of the polymerization and characterization experiments. All authors contributed to editing the manuscript.

Competing interests: The authors declare the following competing financial interests. LPM, GRD, and JSL are inventors on a European patent application (EP19203000.5) on methods for producing the renewable monomer and polymer described here. GRD and JSL are inventors on a European patent application (EP19202957.7) on methods for producing fragments of lignin with functional groups. JSL is an inventor on a European patent application (EP16165180.7) on methods for producing lignin monomers from lignocellulosic biomass during biomass depolymerization. JSL is also a co-founder of Bloom Biorenewables Ltd., which is exploring commercial opportunities for aldehyde-stabilized lignin and aldehydeprotected xyloses.

Data and materials availability: All data needed to support the findings of this study are included in the main text or in the supplementary information. Any additional data can be requested from the corresponding author, JSL.

\section{Supplementary Materials}

Materials and Methods

Supplementary Text

Figs. S1 to S31

Tables S1 to S20

References (35-70) 